\title{
航空发动机轮盘模拟件裂纹预制分析与试验研究
}

\author{
刘若楠 $^{1}$ 耿 $\quad$ 佳 $^{1} \quad$ 张瑞玲 $^{2}$ 陈雪峰 ${ }^{1}$ \\ (1. 西安交通大学机械制造系统工程国家重点实验室 西安 710049; \\ 2. 中航动力股份有限公司强度室 西安 710021)
}

\begin{abstract}
摘要: 航空事故数据显示, 航空发动机轮盘运行可靠性已成为飞行安全的决定性因素之一, 及时有效的发现并排除轮盘结构 缺陷有着非常重大的实际价值。为了给轮盘损伤识别研究提供必要基础, 提出一种可行的轮盘结构裂纹预制方法。为此, 首 先对轮盘模拟件中 $\mathrm{V}$ 型缺口根部应力奇异性分布特征进行了理论分析; 其次, 根据载荷条件和几何特征提出载荷等效方法, 并进行了应力状态及疲劳分析。为了验证所提裂纹预制方法的可行性, 对预制模型进行疲劳试验研究。试验结果显示, 相应 位置处 $x$ 方向应力和疲劳循环载荷次数相对误差约为 $2.7 \%$ 和 $12.7 \%$ 。同时证实所采取的裂纹预制与相应数值分析方法可以应 用于航空发动机轮盘损伤识别方法的研究中。
\end{abstract}

关键词: 轮盘裂纹预制；载荷等效；有限元；应力分析；疲劳分析；裂纹疲劳试验

中图分类号: V232

\section{Theoretical Analysis and Experiment Research for Prefabricating Crack in the Aero-engine Disk Simulator}

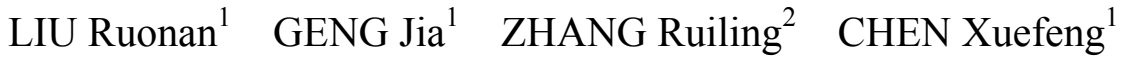 \\ (1. State Key Laboratory for Manufacturing System, Xi' an Jiaotong University, Xi' an 710049; \\ 2. Department of Strength Research, Company Limited of Aviation Power of China, Xi'an 710021)
}

\begin{abstract}
Data of aviation accidents shows that the operation reliability of aero-engine disks has been one of the determining factors for the safe flight of airplane. Therefore, it is valuable and important to diagnosis the disk structural defects in time. To provide the necessary foundation for damage identification of disks, a feasible prefabricating crack method for disk structure is proposed. Firstly, the singularity distribution characteristics in root stress of the V notch in the disk simulator are analyzed in theory sufficiently. Secondly, an equivalent load method is proposed based on the loading conditions and geometrical features. The stress condition and fatigue life are also analyzed. Finally, to verify the feasibility of the proposed method for prefabricating crack, a fatigue experiment is carried on. The experiment results show that the relative errors of stress in $x$ direction and the number of fatigue loading cycles are $2.7 \%$ and $12.7 \%$ respectively, which demonstrate the proposed method for prefabricating crack and corresponding numerical analysis method can be used for damage identification of aero-engine turbine disks.
\end{abstract}

Key words: prefabrication of disk crack; equivalent loading method; finite element analysis; stress analysis; fatigue analysis; fatigue crack experiment

\section{0 前言}

随着世界发达国家航空航天领域对高性能、高 推重比发动机系统和可靠性需求的不断提升 ${ }^{[1,2]}$, 轮 盘作为其关键组件所承受的应力水平和运行安全要 求也随之严苛 ${ }^{[3]}$ 。作为航空发动机的热端部件, 轮 盘需在高温、高转速等极其苛刻的条件下持续运转。

* 国家重点基础研究发展计划(973 计划, 2015CB057400)和国家自然科学 基金(51705397)资助项目。20180722 收到初稿, 20190301 收到修改稿
并且其是航空发动机正常、安全运行的关键零部 件 ${ }^{[4]}$ 。然而, 在实际运行过程中, 持续的高转速致 使轮盘连续承受异常高的离心加速度, 从而使其具 有非常高的应力幅值, 尤其燃气高温同时会降低轮 盘材料自身强度 ${ }^{[5]}$ 。在此条件下，一旦轮盘出现裂 纹缺陷, 其缺陷将快速扩展, 致使轮盘突然破裂, 发动机在短时间内失效, 从而造成重大飞行事故。 据统计, 45\%的航空事故是由于浴轮百故障造成的。 涡轮泵的关键零件就是轮盘, 因此对轮盘裂纹缺陷 快速准确检测具有非常重要的实际意义, 也是未来 
航空航天发展必不可少的应用技术 ${ }^{[6-7]}$ 。近年来, 为 了更有效的排除轮盘缺陷带来的安全隐患, 在裂纹 出现早期能够及时准确发现的损伤识别技术受到各 国政府机构和相关研究人员越来越多的关注 ${ }^{[8]}$ 。与 此同时, 在该领域中, 研究者也已对轮盘裂纹相关 问题进行了理论分析和试验研究并取得了部分成 果。其中, 美国国家航空航天局(NASA)从 2003 年 开始开展旋转轮盘裂纹的振动分析试验, 通过对裂 纹产生的应力场进行理论分析, 试验验证, 试验改 进等。其研究结果表明裂纹会使轮盘产生扭曲的应 力场, 从而产生安全隐患 ${ }^{[8]}$ 。澳大利亚国防部 WANG 等 ${ }^{[9-10]}$ 则对轮盘建立 Jeffcott 转子模型, 认为裂纹会 导致附加不平衡量, 并且与转速的平方成正比, 其 将严重影响轮盘的安全运行。唐金等 ${ }^{[11]}$ 采用 LS-DYNA 软件对浴轮机匣轮盘进行包容性研究, 孙伟等 ${ }^{[12]}$ 提出了基于失效区域划分, 结合受力及结 构形式的不同, 分析盘的应力分布进行裂纹位置预 估及分析方法。以上研究说明裂纹缺陷给轮盘安全 运行带来了难于预估的安全隐患, 致使其安全性难 于准确评估。

因此, 能够在早期准确识别裂纹位置及严重程 度具有重要的理论和实际意义。科研工作者根据轮 盘几何特征和运行特点提出基于动态特征的裂纹识 别方法, 而检验损伤识别方法是否可靠需要基于对 含有预制裂纹的轮盘进行试验研究。然而, 进行裂 纹识别算法验证试验时, 首先完成对轮盘结构进 行的裂纹预制, 是试验进行的必要条件。由于实 际轮盘材料具有刚度大, 造价高等特点, 使得利 用航空航天材料预制裂纹具有非常高的难度，同 时不适用于需要多次进行的试验研究。因此, 本 文根据轮盘几何特征和工作特点利用 Q45 钢制作 了轮盘模拟件，并设计制作了特定尺寸的 $\mathrm{V}$ 型缺 口, 用以最大化的提高局部应力幅值并保证试验 的方便性。从而提高裂纹预制成功率, 降低裂纹 预制试验成本。

为此, 本文首先对模拟件在载荷作用下应力奇 异性进行了充分的理论分析, 并根据实际轮盘模拟 件的几何和加载条件提出了相应的模型简化方法和 载荷等效手段。其次, 在此基础上对该模拟件 $\mathrm{V}$ 型 缺口根部应力场和疲劳循环载荷周期数进行了数值 分析, 并给出了相应应力幅值和疲劳循环数的具体 数值。最后, 为了验证应力场和循环载荷次数结果 数值分析结果与所提裂纹预制方法的可行性, 本文 开展了裂纹预制的试验研究, 并将试验所测应力幅 值和出现裂纹时的循环载荷次数与数值分析结果进 行对比。

\section{1 发动机轮盘模拟件裂纹预制分析}

由于实际航空发动机轮盘制作材料造价和刚度 过高，致使直接用该轮盘进行裂纹预制试验需要过 多的人力物力, 并且绝大多数 ${ }^{[13]}$ 轮盘损伤识别算法 是基于结构运动特征进行, 与材料参数无关。因此 本文根据实际轮盘几何特征, 利用 Q45 钢设计并制 造了轮盘模拟件以此来降低轮盘刚度和造价, 降低 裂纹预制难度，适合于需要进行大量多次的裂纹试 验研究。具体轮盘模拟件工程图如图 1 所示。为了 在有限试验条件下提高试验效率，在模拟件中根据 奇异性分析结果设计并制作了 V 型缺口。

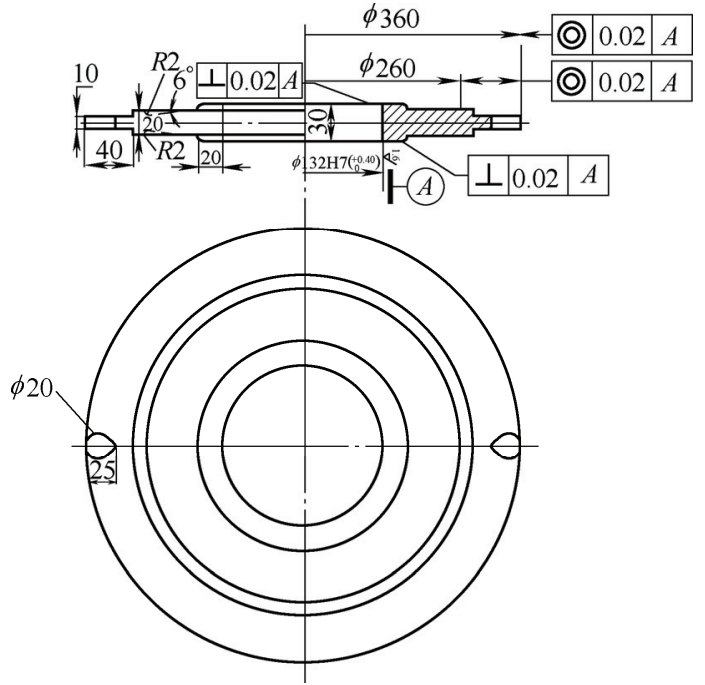

图 1 轮盘模拟件的设计图纸

在应用该模拟件进行裂纹预制试验之前, 需要 对该航空发动机轮盘模拟件的裂纹预制进行必要 的理论分析和数值分析。其中理论分析主要包括判 定应力类型和 $\mathrm{V}$ 型缺口根部应力场奇异性确定。 数值分析则是建立含有奇异单元的二维有限元模 型, 从而实现 $\mathrm{V}$ 型缺口局部应力场和疲劳载荷周 期数分析。

\section{1 理论分析}

如图 2 所示，为轮盘模拟件在预制裂纹时的受 载示意图, 其中 $F_{1}$ 为主动力, 作用于 $\mathrm{V}$ 型缺口一 侧, 其载荷范围为 0 到 $39200 \mathrm{~N}, F_{2}$ 为约束反力, 作用于 $\mathrm{V}$ 型缺口另一侧, 具体如图 2 所示。由于夹 持浴轮盘的工装主要约束其左右移动, 所以 $F_{1}$ 和 $F_{2}$ 在加载过程中存在自动平衡机制。从而使得 $F_{1}$ 和 $F_{2}$ 为大小相等方向相反的两个作用力, 且同时作 用于浴轮盘模拟件。为了进一步说明上述载荷分析 的正确性, 有如图 2 所示工况进行静力学分析。其 中 $M_{0}$ 为约束弯矩, 根据上述分析可知, $M_{0}$ 非常小 
可以不计入应力分析。

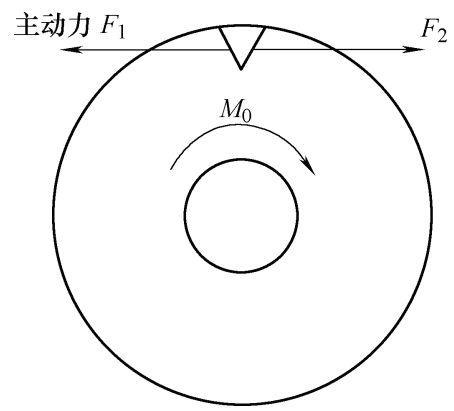

图 2 轮盘受载状态

为此, 根据载荷及约束条件建立力矩平衡方程, 则有

$$
F_{1} \times R-F_{2} \times R-M_{0}=0
$$

式中, $R$ 为载荷位置距离夹持位置处的垂直距离, 根据夹持条件, 中心固定处的约束主要限制其平动, 抗弯矩能力很薄弱, 即 $M_{0} \ll F_{1} \times R$ 。所以, 式(1) 可改写为

$$
F_{1} \times R-F_{2} \times R=0
$$

则 $F_{1}=F_{2}$, 即 $F_{2}$ 和 $F_{1}$ 具有相同的特征, 该理论分析 结果表明上述对模型载荷特征分析结论是正确的。 本文中的试验载荷为面载荷, 面载荷压强大小计算 如下

$$
p=\frac{F_{1}}{A}
$$

式中, $A$ 为载荷作用面的面积。由此可知, 载荷作 用的主要形式为拉, 且为单向载荷形式, 据此可知 浴轮盘的应力状态主要是拉应力, 根据固体力学疲 劳分析准则可知, 本试验的疲劳分析应力应为与载 荷方向相同的拉应力。因此，本文在数值分析中提 取与载荷方向的应力幅值进行疲劳分析研究, 与此 同时为了便于对比数值计算结果和实际测量值，在 数值分析中同时提取。

如图 2 所示, 浴轮盘模拟件的 $\mathrm{V}$ 型缺口根部具 有突变的几何特征, 因而根据弹性力学理论确定其 具有一定的奇异性, 从而将出现应力集中效应。这 将致使利用有限元进行数值分析时具有一定的困 难, 为了进一步了解该 $\mathrm{V}$ 型缺口局部应力场的基本 特征，本文根据文献[14]对其应力奇异性进行了充 分的理论分析。

如图 3 所示, 为一 $\mathrm{V}$ 型缺口在三种载荷作用下 的开裂示意图 ${ }^{[14]}$ 。根据本试验的加载条件, 可以确 定本试验中 $\mathrm{V}$ 型缺口的开裂方式为 $\mathrm{I}$ 型。即，该部 分的理论分析是基于弧开型加载方式进行。该 $\mathrm{V}$ 型 缺口在该载荷类型作用下根部应力状态在笛卡儿坐 标系下的解析表达式如式(4) (6)所示 ${ }^{[14]}$

$$
\begin{gathered}
\sigma_{x}=r^{a_{1}-1}\left[-\frac{2 \alpha_{1}-1-a_{1}}{\left(a_{1}-1\right) \alpha_{1}} \cos \left(a_{1}-1\right) \theta+\cos \left(a_{1}-3\right) \theta\right]\left[-a_{1}\left(a_{1}-1\right) \alpha_{1}\right] a_{11}+ \\
r^{a_{2}-1}\left[-\frac{2 \beta_{2}-1-a_{2}}{\left(a_{2}-1\right) \alpha_{1}} \sin \left(a_{2}-1\right) \theta+\sin \left(a_{2}-3\right) \theta\right]\left[-a_{2}\left(a_{2}-1\right) \beta_{2}\right] b_{21} \\
\sigma_{y}=r^{a_{1}-1}\left[-\frac{2 \alpha_{1}+1+a_{1}}{\left(a_{1}-1\right) \alpha_{1}} \cos \left(a_{1}-1\right) \theta-\cos \left(a_{1}-3\right) \theta\right]\left[-a_{1}\left(a_{1}-1\right) \alpha_{1}\right] a_{11}+ \\
r^{a_{2}-1}\left[-\frac{2 \beta_{2}+1+a_{2}}{\left(a_{2}-1\right) \beta_{2}} \sin \left(a_{2}-1\right) \theta-\sin \left(a_{2}-3\right) \theta\right]\left[-a_{2}\left(a_{2}-1\right) \beta_{2}\right] b_{21} \\
\tau_{x y}=r^{a_{1}-1}\left[-\frac{a_{1}+1}{\left(a_{1}-1\right) \alpha_{1}} \sin \left(a_{1}-1\right) \theta-\sin \left(a_{1}-3\right) \theta\right]\left[-a_{1}\left(a_{1}-1\right) \alpha_{1}\right] a_{11}+ \\
r^{a_{2}-1}\left[-\frac{a_{2}+1}{\left(a_{2}-1\right) \beta_{2}} \cos \left(a_{2}-1\right) \theta+\cos \left(a_{2}-3\right) \theta\right]\left[-a_{2}\left(a_{2}-1\right) \beta_{2}\right] b_{21}
\end{gathered}
$$

式中, 参数 $a_{1}$ 和 $a_{2}$ 均由 $\mathrm{V}$ 型缺口的张开角度 $\theta_{0}$ 决定, 具体参见文献 $[14]$ 。据式(4) (6), V 型缺 口根部的应力奇异性由 $a_{1}$ 和 $a_{2}$ 同时决定。当其值 小于 1 时, 值越小奇异性越大。本文以 $\alpha=360-\theta_{0}$ 来进行表征, 从而得到 $\alpha$ 与 $a_{1}$ 和 $a_{2}$ 值大小之间的 关系趋势如图 4 所示 ${ }^{[14]}$ 。由图可以看出, 当 $\alpha=$ $360^{\circ}$ 时, 奇异性是最大的, 此时 $\mathrm{V}$ 型缺口变化为 线型裂纹。
据此，结合试验可操作性设计了如图 4 所示带 有 $\mathrm{V}$ 型缺口的轮盘模拟件。其开口角度 $\theta_{0}$ 为 $33.7^{\circ}$, 因此 $\alpha=326.3^{\circ}$ 。由图 3 可以看出 $a_{1}$ 约为 $0.5, a_{2}$ 约 为 0.8 。其根部应力奇异性逼近于线性裂纹, 几乎进 入如图 5 所示的奇异性关系曲线的水平区域, 尤其 是 $a_{1}$ 已经进入水平区域，因此本文采用线性裂纹模 型进行有限元数值分析。这将大大降低数值分析成 本，简化分析难度。 
$\tau_{y z}$ (III型，撕开型加载

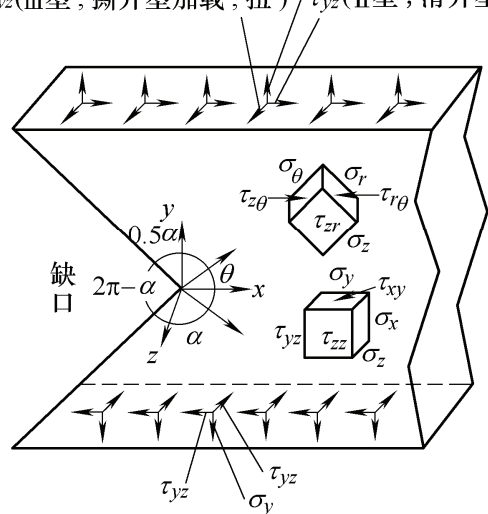

图 $3 \mathrm{~V}$ 型缺口开裂示意图

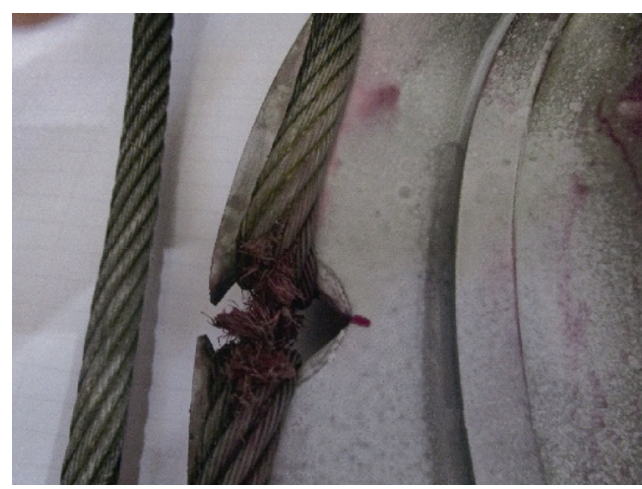

图 4 轮盘受载实物图

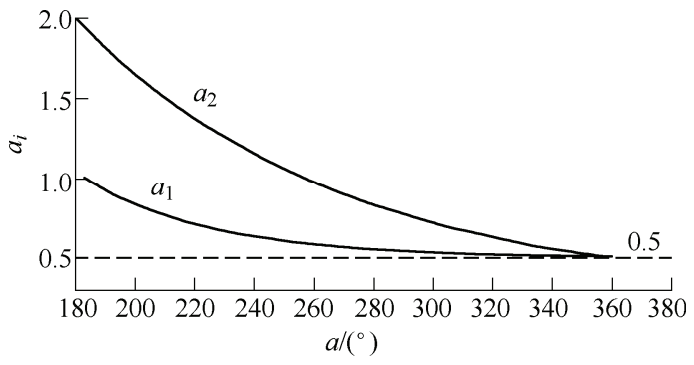

图 $5 \alpha$ 角与奇异性的关系

\section{2 数值分析}

基于上述理论分析结果, 本文利用 ANSYS 12.0 有限元平台中的二维奇异单元模拟 $\mathrm{V}$ 型缺口根部的 奇异性特征，利用分析线形裂纹的方法反映 $\mathrm{V}$ 型缺 口根部的应力状态。数值分析所采用材料的物理和 力学参数如表 1 所示, 其中 $\sigma_{\mathrm{s}}$ 为屈服极限, $\sigma_{\mathrm{b}}$ 为破 断极限。数值分析部分主要进行了轮盘结构第一主 应力、 $x$ 方向的应力和疲劳数值分析, 用以确认试 验安全性和疲劳裂纹出现时的循环载荷次数。

表 1 模拟件的材料参数及强度参数

\begin{tabular}{ccccc}
\hline 弹性模量 $/ \mathrm{GPa}$ & 泊松比 & 密度 $/\left(\mathrm{kg} \cdot \mathrm{m}^{-3}\right)$ & $\sigma_{\mathrm{s}} / \mathrm{MPa}$ & $\sigma_{\mathrm{b}} / \mathrm{MPa}$ \\
\hline 206 & 0.26 & 7860 & 355 & 600 \\
\hline
\end{tabular}

为了分析轮盘模拟件在实际加载作用下(图 3) 的应力状态, 用以确保模拟件在试验过程中不会出 现突发性破断。由于在 $\mathrm{V}$ 型缺口两侧的载荷类型为
面载荷, 故本文建立如图 $6 \mathrm{a}$ 和 $6 \mathrm{~b}$ 所示 $\mathrm{V}$ 型缺口和 线型裂纹下的载荷分析模型。利用 $\mathrm{V}$ 型缺口和线性 裂纹共同等效实际模拟件的 $\mathrm{V}$ 型缺口。图 $6 \mathrm{a}$ 和 $6 \mathrm{~b}$ 中斜面简化图用来指示实际 $\mathrm{V}$ 型缺口和等效 $\mathrm{V}$ 型缺 口张开角度特征，很显然在等效过程中张开角度特 征发生了变化。由于该斜度直接影响等效载荷大小, 本文将其单独列出得到如图 $7 \mathrm{a}$ 和 $7 \mathrm{~b}$ 所示直角三角 形, 令图中所示三角形角度分别为 $\theta_{1}$ 和 $\theta_{2}$ 。其中原 有 $\mathrm{V}$ 型缺口和等效线型裂纹模型的简化图分别为图 $6 \mathrm{a}$ 和 $7 \mathrm{~b}$ 。由图中可以看出, 线型裂纹的加载斜度低 于 $\mathrm{V}$ 型缺口。所以，需对线型裂纹的加载进行重新 分析, 即进行等效处理。因此, 为了便于利用 ANSYS 12.0 自带奇异单元进行数值分析, 需要对该 $\mathrm{V}$ 型缺 口的几何和载荷特征进行等效修正，使之符合奇异 单元的使用要求, 并且保证与实际加载效果一致, 即改变模型几何条件后的有限元分析模型的加载载 荷对 $\mathrm{V}$ 型缺口根部施加的弯矩载荷和拉载荷能够与 实际载荷相一致。

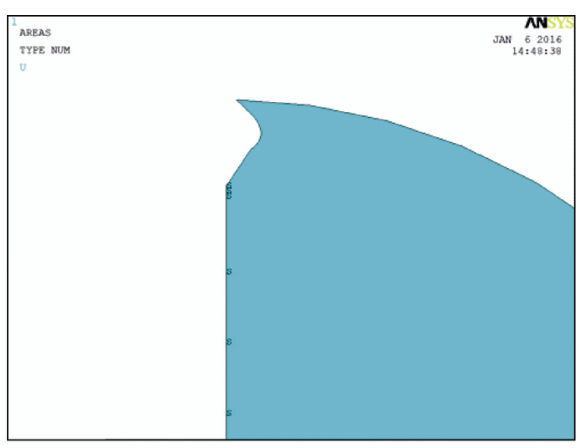

（a）测量 V 型缺口的几何特征

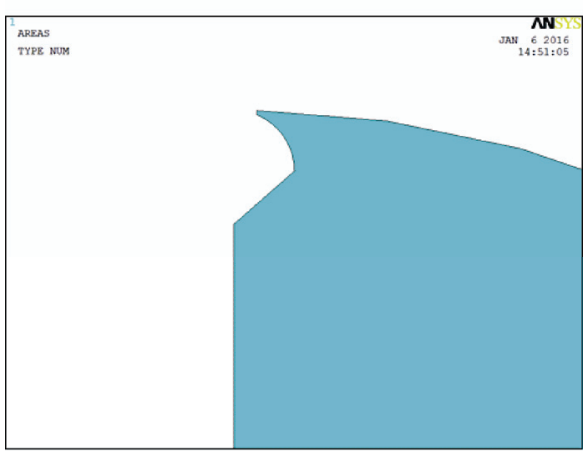

(b) 等效线型裂纹几何特征

图 $6 \mathrm{~V}$ 型缺口简化模型

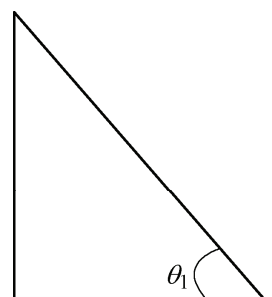

(a) $\mathrm{V}$ 型缺口

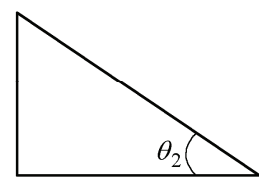

(b) 线型裂纹
图 $7 \mathrm{~V}$ 型缺口和线型裂纹载荷分析 
根据测量结果计算可得 $\theta_{1}=73.15^{\circ}, \theta_{2}=41.29^{\circ}$ 。 根据理论力学力平移法则和力矩平衡, 计算可得加 载于实际 $\mathrm{V}$ 型缺口侧面的压力等效到根部的力载荷 和弯矩载荷, 分别记为 $F_{V x} 、 F_{V y}$ 和 $M_{v}$ 。其具体数值 $F_{V x} 、 F_{V y}$ 和 $M_{v}$ 分别为 $2.2125 \times 10^{4} \mathrm{~N}, 6.7011 \times 10^{3} \mathrm{~N}$, $199 \mathrm{~N} \cdot \mathrm{m}$ 。

为了计算有限元分析模型的等效载荷在斜面的 具体加载位置和大小, 令等效载荷为 $F_{l x}=F_{V x}$ 和 $F_{l y}=$ $F_{V y}$ 。并建立如下式力矩平衡方程, 确定加载位置

$$
F_{l x}(x+0.00625 \mathrm{~m})+\frac{F_{l x}}{\tan \theta_{2}}=199 \mathrm{~N} \cdot \mathrm{m}
$$

其中 $x$ 为表征载荷位置的特征量, 求得 $x$ 为 $0.002 \mathrm{~m}$ 。 该载荷特征将被计入有限元分析中, 将实际载荷进 行等效处理。

由于进行静载拉伸试验时, 受载部位的应力变 形条件与应力状态比较简单。据此建立轮盘等效线 型二维有限元分析模型, 具体如图 8 所示, 其中具 体的加载条件严格按照上述计算结果进行加载。在 垂直对称轴位置处施加对称约束, 在中心圆柱孔处 施加全约束。经 ANSYS 12.0 计算得到裂纹根部区 域内第一主应力和 $x$ 方向应力云图如图 9a、 $9 b$ 所示。 $\mathrm{V}$ 型缺口根部区域可测部位的第一主应力和 $x$ 方向 应力最大值约为 $430 \mathrm{MPa}$ 和 $401 \mathrm{MPa}$ 。

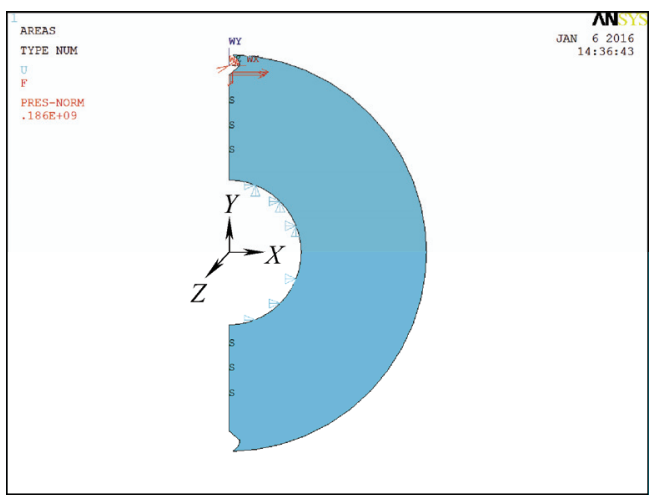

图 8 轮盘模拟件有限元分析模型

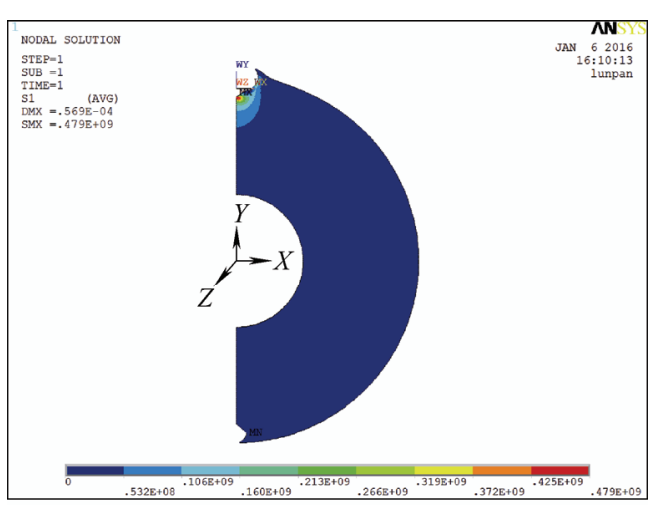

(a) 第一主应力云图

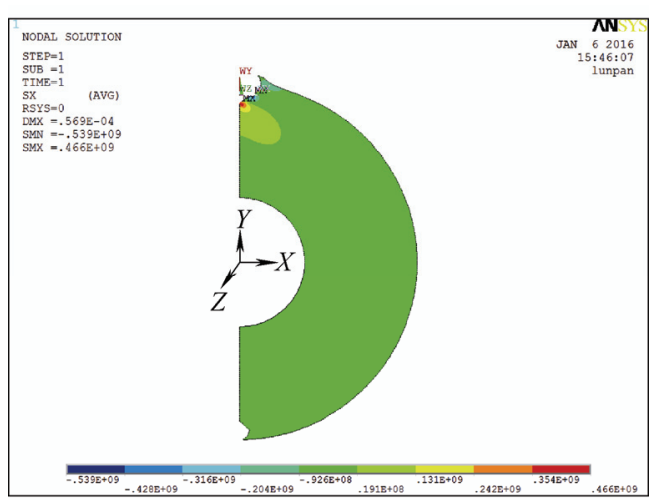

(b) $x$ 方向应力云图

图 9 轮盘模拟件应力云图

对于标准试样, 反映外加循环应力 $S$ 和疲劳寿 命 $N$ 之间关系的曲线叫做应力-寿命曲线, 也称 $S-N$ 曲线。一般可假设材料的 $S-N$ 试验数据服从单对数 线性关系 ${ }^{[15]}$, 即

$$
\Delta \sigma=m \lg N+A
$$

式中, $m, A$ 为常数, 需要通过材料的疲劳性能试验 获得。对于 45 钢, 其 $S-N$ 曲线模型采用下式

$$
\Delta \sigma_{\text {int }}=-75 \lg N+725
$$

式中, $\Delta \sigma_{\text {int }}$ 为应力指标幅值, 单位为 $\mathrm{MPa} 。 N$ 为循 环次数。将有限元计算第一主应力幅值 $430 \mathrm{MPa}$ 代 入式(9)中可得 $N$ 约为 8500 次。

\section{2 轮盘裂纹预制试验研究}

为了验证上述理论和数值分析研究结果的合理 性, 进行了轮盘裂纹预制的试验研究。其主要内容 为测量 $\mathrm{V}$ 型缺口根部 $x$ 方向的应力和疲劳裂纹出现 时的循环载荷次数, 并将结果与数值分析结果进行 对比。

\section{1 轮盘模拟件试验应力分析}

为了验证本文对于轮盘模拟件裂纹尖端应力状 态的计算结果，对轮盘模拟件在载荷作用下的 $x$ 方 向应力进行测量。如图 10 所示为测量轮盘 $\mathrm{V}$ 型缺 口根部附近 $x$ 方向应力时的试验示意图, 将电阻应

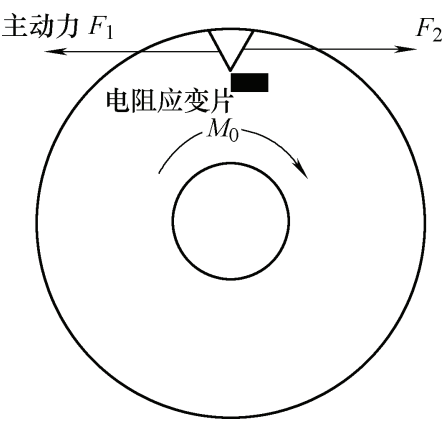

图 10 电阻应变片的安装位置 
变片贴在缺口尖端, 利用桥式电路对裂纹尖端应力 进行测量。

利用液压系统调节装置将主动加载力 $F_{1}$ 设定 为梯度上升, 分别为 $8820 \mathrm{~N}, 17640 \mathrm{~N}, 26460 \mathrm{~N}$, $35280 \mathrm{~N}, 38220 \mathrm{~N}$, 主动力加载剖面图如图 11 所 示。测量得电阻应变片的微应变与相应的应力值如 表 2 所示。根据表格可以看出, 试验结果与有限元 计算的 $x$ 方向的应力值 $401 \mathrm{MPa}$ 结果非常匹配, 计 算误差约为 $2.7 \%$ 。可见计算结果与实测值也已经非 常接近, 精度非常高。说明本文所使用的数值分析 方法对该轮盘模拟件在如图 5 所示载荷作用下的应 力幅值具有很好的求解能力。所以, 本文根据实际 工况所提出的模型简化和载荷等效方法具有很好的 求解精度。

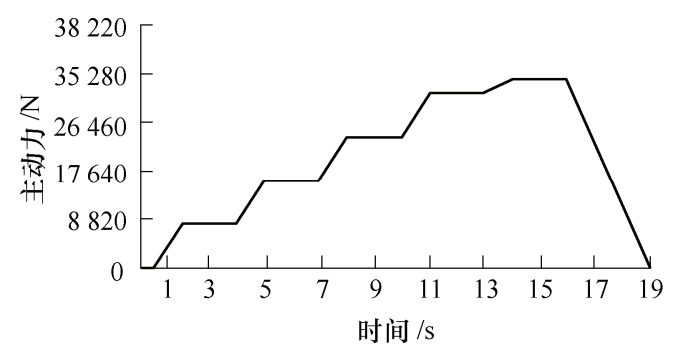

图 11 主动力加载剖面图

表 2 电阻应变片所测微应变与应力值

\begin{tabular}{ccc}
\hline 主动力 $F_{1} / \mathrm{N}$ & 微应变 $\mu / \times 10^{-6}$ & $x$ 方向应力值 $/ \mathrm{MPa}$ \\
\hline 8820 & 350.74 & 72.25 \\
17640 & 750.12 & 154.52 \\
26460 & 1166.03 & 240.20 \\
35280 & 1641.52 & 338.15 \\
38220 & 1875.67 & 386.39 \\
\hline
\end{tabular}

\section{2 轮盘模拟件试验应力分析}

由于低循环疲劳失效是浴轮盘的主要失效模 式 ${ }^{[16-17]}$, 并且疲劳寿命具有较大的分散性, 因此本 试验使用液压式疲劳拉伸试验机对轮盘模拟件进行 疲劳拉伸试验, 用以进行预制裂纹并验证数值分析 的裂纹出现时的循环载荷次数。本试验确定的加载 循环载荷大小为 $38220 \mathrm{~N}, 14 \mathrm{~s}$ 为一个循环。

在 7400 次循环的时候目视未发现裂纹, 7420 次循环时发现裂纹, 经过荧光探伤判定裂纹已经出 现。即在 20 次循环内轮盘模拟件 V 型缺口根部出 现线型穿透性裂纹, 具体如图 12 所示。其中紫色线 条记为裂纹位置, 线条长度表示裂纹长度, 经过测 量其径向长度约为 $3 \mathrm{~mm}$ 。

由于出现裂纹时的循环载荷次数约为 7420 次, 在疲劳分析领域内, 这一结果与数值分析结果的 8500 次已相当接近, 相对误差约为 $12.7 \%$ 。说明本 文所提疲劳分析和应力分析方法精度相当高, 具有

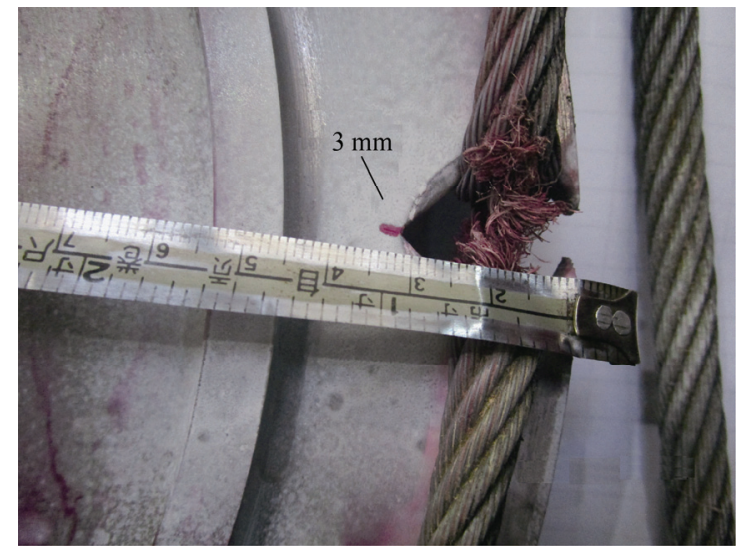

图 12 拉裂的轮盘模拟件

很好的可操作性。并且由于应力考核时应变片测得 应力并非裂纹尖端应力, 测量值必定会小于最大应 力, 因此使用 $S-N$ 曲线所匹配的循环次数 $N$ 是实际 循环次数的上限。即实际裂纹产生所需的循环数应 小于之前的计算值, 这也与试验结果相吻合。因此 本文采用的线型裂纹计算 $\mathrm{V}$ 型缺口应力状态以及疲 劳分析的方法具有一定的说服力, 结果可以用以指 导裂纹预制试验。

\section{3 结论}

(1) V 型缺口由于其奇异性, 使得受力计算普遍 误差大, 而本文针对轮盘缺口根部应力分布特征提 出的裂纹等效模型和载荷等效方法可以较为准确计 算出其根部应力值的具体分布。

（2）与其他预制裂纹的方法相比，本文提出的 设计 $\mathrm{V}$ 型缺口预制轮盘裂纹能够更有效地模拟轮盘 裂纹, 便于试验操作, 并优化获取最大应力值, 同 时理论和数值分析结果显示对其进行相应等效处理 可以获取很好的应力和疲劳计算结果。

(3) 试验结果显示理论分析结果与试验结果基 本一致, 同时也较为准确地预测了裂纹出现时的循 环载荷周期数。因此本文所提裂纹预制和应力疲劳 分析方法非常有效, 能够为轮盘损伤识别研究与裂 纹预制试验提供完整且有效的参考依据。

\section{参 考 文 献}

[1] 巩孟林, 陈卫, 钟也磐, 等. 基于稀疏表示和 SVM 的 航空发动机故障诊断[J]. 噪声与振动控制, 2017(3): 162-167.

GONG Menglin, CHEN Wei, ZHONG Yepan, et al. Fault diagnosis of aircraft engines based on sparse representation and SVM [J]. Noise and Vibration Control, 2017(3): 162-167.

[2] LIU Ruonan, YANG Boyuan, ZHANG Xiaoli, et al. 
Time-frequency atoms-driven support vector machine method for bearings incipient fault diagnosis[J]. Mechanical Systems and Signal Processing, 2016, 75: 345-370

[3] 李宏新, 王延荣, 程域钊. 基于归一化参数模型的涡轮 盘和浴轮叶片蠕变分析 [J]. 航空发动机, 2016(5): 48-54.

LI Hongxin, WANG Yanrong, CHENG Yuzhao. Analysis of creep based on normalized-parameter model for turbine disk and blade [J]. Aeroengine, 2016(5): 48-54.

[4] FU Youzhi, GAO Hang, WANG Xuanping, et al. Machining the integral impeller and blisk of aero-engines : A review of surface finishing and strengthening technologies[J]. Chinese Journal of Mechanical Engineering, 2017，30(3): 528-543.

[5] 赵荣国, 刘亚风, 蒋永洲, 等. 航空发动机涡轮盘用 GH4133B 合金疲劳裂纹扩展寿命概率预测 [J]. 机械工 程学报，2015，51(18): 71-82.

ZHAO Rongguo, LIU Yafeng, JIANG Yongzhou, et al. Probabilistic fatigue crack propagation life prediction of GH4133B superalloy used in turbine disk of aero-engine [J]. Journal of Mechanical Engineering, 2015, 51(18): 71-82.

[6] 赵荣国, 李红超, 蒋永洲, 等. 航空发动机浴轮盘用 GH4133B 合金疲劳裂纹萌生与短裂纹扩展研究[J]. 机 械工程学报, 2013，49(10): 116-126.

ZHAO Rongguo, LI Hongchao, JIANG Yongzhou, et al. Research on fatigue crack initiation and short crack propagation of GH4133B superalloy used in turbine disk of aero-engine [J]. Journal of Mechanical Engineering, 2013, 49(10): 116-126.

[7] 张智轩, 石多奇, 杨晓光. 含销钉孔边裂纹的某压气机 轮盘裂纹扩展分析 [J]. 航空动力学报, 2016(3): 567-574.

ZHANG Zhixuan, SHI Duoqi, YANG Xiaoguang. Analysis of crack propagation for a compressor disk with cracks on pin holes [J]. Journal of Aerospace Power, 2016 (3): 567-574.

[8] WOIKE M R, ABDUL A A. Crack-detection experiments on simulated turbine engine disks in NASA glenn research center's rotordynamics laboratory[J]. NASA TM, 2010, 216239.

[9] WANG W. Disk crack detection and diagnosis for gas turbine engines $[\mathrm{C}] / /$ Aerospace Conference, 2006 IEEE. IEEE, 2006: 12.

[10] WANG W, MUSCHLITZ G. Disk crack detection in spin testing using tip timing data[C]//Aerospace Conference, 2010 IEEE. IEEE，2010: 1-14.

[11] 唐金, 唐广, 宣海军, 等. 航空发动机涡轮机匣轮盘包 容性研究[J]. 航空动力学报, 2016(6): 1393-1399.

TANG Jin, TANG Guang, XUAN Haijun, et al. Investigation on disk containment of aero-engine turbine casing[J]. Journal of Aerospace Power, 2016(6) : 1393-1399.

[12] 孙伟, 朱晔, 霍军周, 等. 基于裂纹失效区域划分的 TBM 刀盘裂纹位置预估及分析 [J]. 机械工程学报, 2018, 54(1): 27-35.

SUN Wei, ZHU Ye, HUO Junzhou, et al. Predict and analysis the locations of fatigue crack in TBM cutterhead based on crack failure [D]. Journal of Mechanical Engineering, 2018, 54(1): 27-35.

[13] 黄燕晓, 李书明, 藏雅霖. 基于模态参数的航空发动机 浴轮盘结构损伤检测 [J]. 航空维修与工程, 2009(6): 78-80.

HUANG Yanxiao, LI Shuming, ZANG Yalin. The structure damage detection on aero-engine turbine disc based on modal parameters [J]. Aviation Maintenance and Engineering, 2009(6): 78-80.

[14] 涂传林. 混凝土坝坝踵区的断裂分析 [J]. 水利学报, 1981(4): 38-50.

TU Chuanlin. Fracture analysis on the concrete dam heel [J]. Journal of Hydraulic Engineering, 1981(4): 38-50.

[15］曾攀，雷丽萍，方刚. 基于 ANSYS 平台有限元分析手 册结构的建模与分析 [M]. 北京: 机械工业出版社, 2010 .

ZENG Pan, LEI Liping, FANG Gang. Finite element analysis guide: Modeling and analysis of structure [M]. Beijing: China Machine Press, 2010.

[16] ZHANG Junhong, YANG Shuo, LIN Jiewei. Fatigue crack growth rate of Ti- $6 \mathrm{Al}-4 \mathrm{~V}$ considering the effects of fracture toughness and crack closure[J]. Chinese Journal of Mechanical Engineering, 2015，28(2): 409-415.

[17] GUO C Z, YAN J H, BERGMAN L A. Experimental dynamic analysis of a breathing cracked rotor[J]. Chinese Journal of Mechanical Engineering, 2017, 30(5) : $1177-1183$

作者简介: 刘若楠, 女, 1990 年出生, 博士研究生。主要研究方向为故 障诊断。

E-mail: liuruonan04@163.com

陈雪峰(通信作者), 男, 1975 年出生, 博士, 教授, 博士研究生导师。 主要研究方向为故障诊断, 振动分析与有限元分析。

E-mail: chenxf@mail.xjtu.edu.cn 